\title{
Testicular Choriocarcinoma with Inexplicably High Beta-human Chorionic Gonadotropin and Renal Metastases
}

\author{
Jatin Sarin, ${ }^{1}$ Rimpi Bansal, ${ }^{1}$ Puneet Kaur, ${ }^{2}$ Anureet Kaur, ${ }^{3}$ and Akshita Sarin ${ }^{4}$ \\ 1. Chandigarh Cancer and Diagnostic Centre, Chandigarh, India; 2. Indus Superspeciality Hospital, Punjab, India; 3. Gian Sagar Medical College and \\ Hospital, Punjab, India; 4. Government Medical College and Hospital, Chandigarh, India
}

DOI: https://doi.org/10.17925/OHR.2021.16.2.124

$\mathrm{C}$ horiocarcinoma of testes is a very rare tumor, occurring either in pure form or as part of mixed germ cell tumor. The primary route of metastasis is hematogenous, as this type of tumor is extremely vascular. Germ cell tumors, otherwise, generally have a propensity for lymphatic spread. The most common presentation is due to symptoms arising from the metastasis of the tumor to sites such as the lungs, liver, gastrointestinal tract, spleen, brain, and adrenal glands. We report this case of a young male with primary testicular choriocarcinoma who had renal metastasis, which was discovered during the process of evaluation. This is a rare site of metastasis, which can result in spontaneous renal hemorrhage and hematuria. The $\beta$-HCG level of our patient was extremely high $(886,270 \mathrm{mIU} / \mathrm{mL})$, which indicates a high tumor load in the body and subsequent poor outcome.

\section{Keywords}

Testicular choriocarcinoma, $\beta$-HCG, renal metastases, seminoma

Disclosure: Jatin Sarin, Rimpi Bansal, Puneet Kaur, Anureet Kaur, and Akshita Sarin have no financial or non-financial relationships or activities to declare in relation to this article.

Review Process: Double-blind peer review.

Compliance with Ethics: Informed consent was received from the patient involved in this case study, and no identifying information or images have been included. Authorship: The named authors meet the Internationa Committee of Medical Journal Editors (ICMJE) criteria for authorship of this manuscript, take responsibility for the integrity of the work as a whole, and have given final approval for the version to be published.

Access: This article is freely accessible at touchONCOLOGY.com @ C Touch Medical Media 2020

Received: March 18, 2020

Accepted: November 14, 2020

Published Online: December 23, 2020

Citation: Oncology \& Hematology Review (US) 2020;16(2):124-6

Corresponding Author: Rimpi Bansal, Chandigarh Cancer and Diagnostic Centre,

SCO No. 23, SECTOR - 33-D, Chandigarh 160020, India. E: rimpijatinsarin@yahoo.com

Support: No funding was received in the publication of this article.
Choriocarcinoma of the testes is very rare, occurring either in pure form or as part of mixed germ cell tumor. ${ }^{1,2}$ Pure choriocarcinoma constitutes $<1 \%$ of choriocarcinoma cases, whereas mixed tumors with choriocarcinoma make up $8 \%$ of testicular germ cell tumors. ${ }^{2}$ The overall incidence of choriocarcinoma is 0.8 cases per 100,000 male population in countries with highest frequency of testicular cancer. ${ }^{2}$ Choriocarcinoma has the propensity to invade blood vessels, which leads to early dissemination of the disease. The most common presentation is due to symptoms arising from the metastases of tumor. Common sites of dissemination are the lungs, liver, gastrointestinal tract, spleen, brain, and adrenal glands. Patients typically have high beta-human chorionic gonadotropin ( $\beta-\mathrm{HCG})$ levels, commonly $>1,000 \mathrm{mIU} / \mathrm{mL} .^{1,2}$

We present the case of a patient with testicular choriocarcinoma with renal metastases and inexplicably high $\beta$-HCG levels, indicating extremely high tumor burden and poor prognosis. We report this case on account of its rare incidence and rarer site of metastases, which has been known to result in spontaneous renal hemorrhage and hematuria in patients with metastatic choriocarcinoma to kidneys. ${ }^{3}$

\section{Case presentation}

A 35-year-old male patient presented with a history of injury to the right testis. He had noticed the unequal size of his testes, with the right one being bulkier than the left. Detailed history revealed that the patient also had a productive cough for 2 months. On evaluation, contrast-enhanced computed tomography (CT) of the chest showed multiple well-defined soft tissue nodules diffusely scattered in both lungs, with mild pleural effusion on the right side. Ultrasonography of the scrotum showed a large heterogeneous mass replacing the right testis. Serum marker study showed: $\beta$-HCG $886,270 \mathrm{mlU} / \mathrm{mL}$, alpha-fetoprotein $1.22 \mathrm{ng} / \mathrm{mL}$, and lactate dehydrogenase (LDH) 2,329 U/L. Baseline two-dimensional echocardiography showed left ventricle ejection fraction was normal at $57 \%$. The patient was classified as high risk according to the International Germ Cell Cancer Collaborative Group criteria. ${ }^{2}$ He underwent right orchiectomy; post-operative scans and the histopathology report of surgical scan are described subsequently.

Post-operative positron emission tomography (PET)-CT scan showed moderate right pleural effusion with basal atelectasis in the lower lobe, and multiple nodular opacities in the parenchyma and pleura (Figure 1). The paratracheal, pre-tracheal, paraaortic, precarinal, subcarinal, bilateral hilar, and supradiaphragmatic lymph nodes were mildly fluorodeoxyglucose (FDG)-avid, with maximum standardized uptake value (SUVmax) of 4.70. An FDG-avid, centrally necrotic, soft-tissue lesion was 


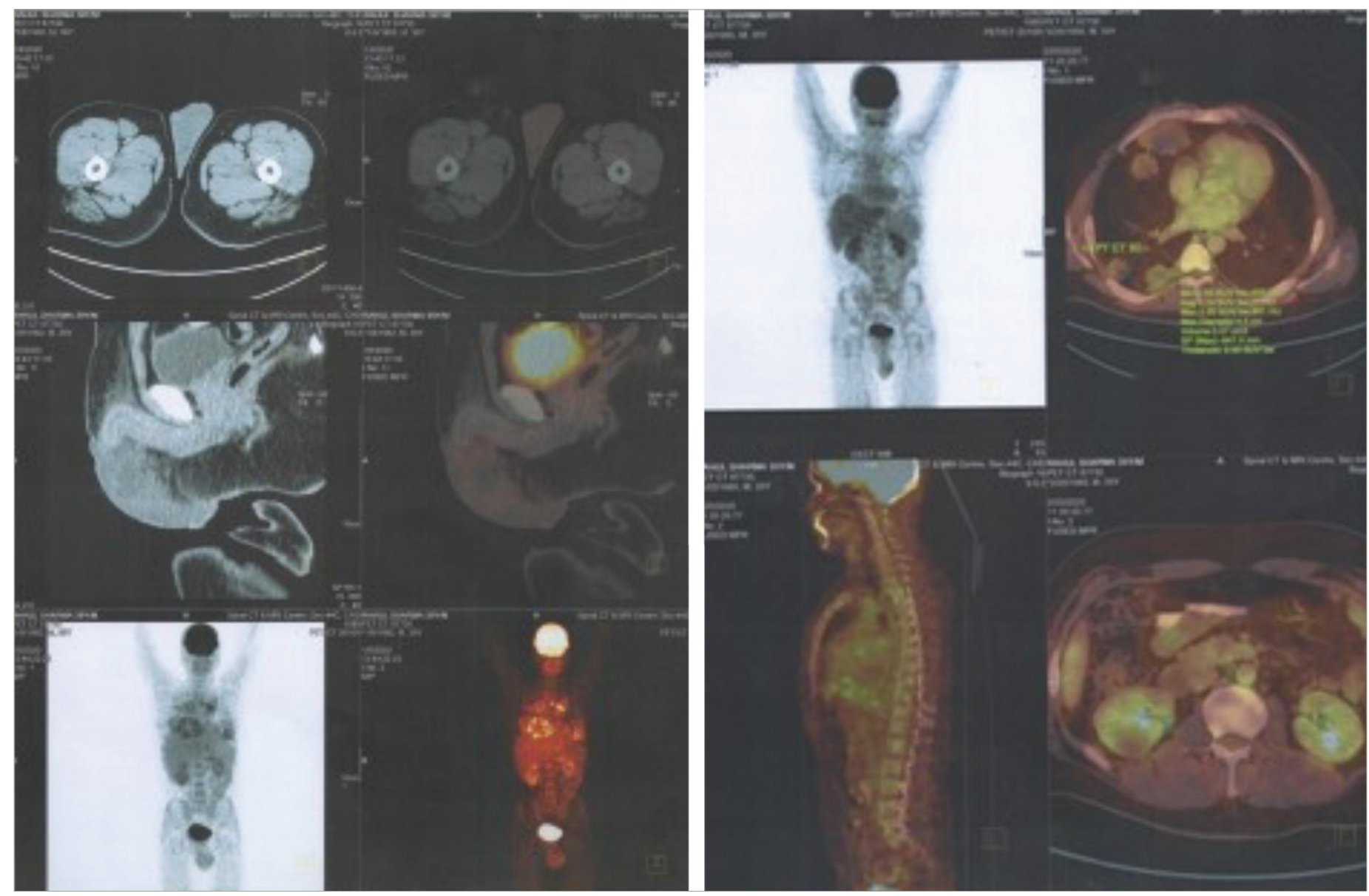

Positron emission tomography-computed tomography scan on the right side shows a well-defined fluorodeoxyglucose-avid standardized uptake value max 4.96 lesion on the lower pole of the right kidney.

detected in the lower pole region of right renal parenchyma, with maximum suVmax of 4.96 , and measuring $3.4 \times 3.0 \times 4.1 \mathrm{~cm}$. The right testicle was not seen post-operatively.

The histopathology report, after right inguinal orchiectomy, showed a tumor measuring $13.0 \times 9.5 \times 9.5 \mathrm{~cm}$ replacing the normal tissue and involving hilar soft tissue. The tumor was reddish-brown in color with areas of hemorrhage and necrosis. Morphological features were of malignant mixed germ cell tumor, consisting predominantly of choriocarcinoma (95\%) with large areas of necrosis and hemorrhage and a minor component of seminoma (5\%).

The patient was initiated on adjuvant chemotherapy regimen comprising of cisplatin, etoposide, and bleomycin (PEB) on a 5-day schedule with standard dosing_-bleomycin $30 \mathrm{mg}$ intravenous bolus on days 2, 9, and 16; etoposide $100 \mathrm{mg} / \mathrm{m}^{2} /$ day for 5 days; and cisplatin $20 \mathrm{mg} / \mathrm{m}^{2} /$ day for 5 days. Follow-up PET-CT scan revealed excellent response after four cycles of BEP. The renal lesion and other metastatic deposits disappeared completely. There were non-FDG-avid lesions in both lungs, the largest one was approximately $4.5 \times 7.0 \mathrm{~cm}$ in the subpleural region of the lower lobe of the right lung. It is possible that these lesions occurred due to fibrosis of necrotic lesions in response to chemotherapy. Both $\beta$-HCG and LDH levels were drastically reduced (Figure 2). The persistent fibrotic nodules in both the lungs were removed surgically. The patient received regular follow-up for 6 months and is doing well.

\section{Discussion}

Testicular cancer is the most common malignancy in men aged 15-34 years and accounts for approximately $1 \%$ of all cancer in men. ${ }^{1,2}$ Choriocarcinoma is an unusual occurrence in testicular tumors, with an incidence of approximately 0.8 cases per year per 100,000 male population in countries with the highest frequency of testicular cancer. ${ }^{2}$ Jiang et al. retrospectively analyzed cases of males with choriocarcinoma treated at Peking Union Medical College Hospital between 1990 and 2012, and found that the mean age of occurrence was 20-29 years. ${ }^{4}$ Choriocarcinoma may be a part of non-seminomatous component or may exist in pure form as part of germ cell tumor. Both instances carry a poor prognosis for the patient. In the case presented here, choriocarcinoma constituted around 95\% of the entire tumor, whilst $5 \%$ was seminoma.

The neoplastic trophoblasts have an affinity for blood vessels, and the hematogenous spread to different organs, such as the lungs, adrenals, liver, and brain appears early in the course of the disease and often results in the first manifested symptom. A series by Alvarado-Cabrero et al. confirms the proclivity for high-stage presentation, including the presence of distant metastasis, hematogenous spread, and poor outcomes related 
Figure 2: Change in serum levels of tumor markers

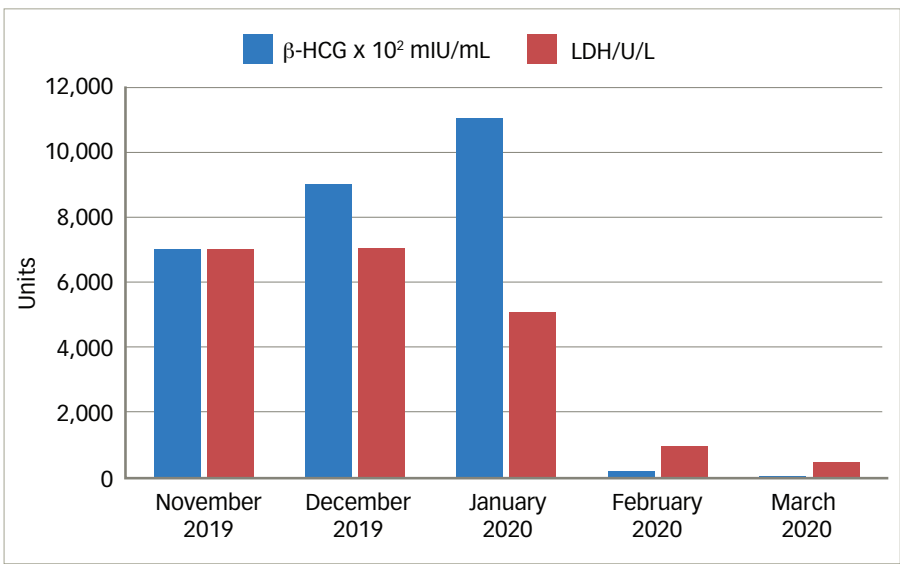

Lactate dehydrogenase and beta-human chorionic gonadotropin ( $\beta-H C G)$ during the course of therapy. $\beta$-HCG level showed a mild increase after surgery. Both tumor marker levels plummeted after the patient received chemotherapy.

$\beta-H C G=$ beta-human chorionic gonadotropin; $L D H=$ lactate dehydrogenase

to testicular choriocarcinoma. Mixed germ cell tumors with a predominant choriocarcinoma component have a similar tendency for high-stage presentation, marked elevation of serum $\beta$-HCG levels, and aggressive behavior compared with pure choriocarcinoma. ${ }^{5}$

An important, and often fatal, complication of choriocarcinoma is due to spontaneous or post-chemotherapeutic hemorrhage. When this occurs in the lungs, the commonest site of metastases, it leads to hypoxic respiratory failure due to diffuse alveolar hemorrhage consequent to necrosis of vascular tumors. ${ }^{6,7}$

Some unusual sites of metastasis reported in the literature are the stomach, duodenum, jejunum, kidney, skin, and neck. Chaar et al. ${ }^{8}$ and Yokoi et al. ${ }^{9}$ have reported cases of metastasis of choriocarcinoma in the jejunum. Lowe et al. presented a case of an ulcerated mass in the stomach, where pathology confirmed it to be a malignant, poorly differentiated choriocarcinoma. ${ }^{10}$ Lauro et al. have described stomach tumor when patients presented with a chief complaint of hematemesis. ${ }^{11}$ Vardaros et al. reported a case of duodenal metastasis from choriocarcinoma, ${ }^{12}$ and Senkal et al. ${ }^{13}$ and Ahmadi et al. ${ }^{14}$ have independently reported cases of neck masses as the primary presentation in cases of testicular choriocarcinoma. Toberer et al. reported a case of cutaneous metastasis from underlying choriocarcinoma, in which the patient had an extremely high disease load and died within 9 days of treatment initiation..$^{15}$ Uveal and choroidal metastases have also been reported in the literature. ${ }^{16,17}$ Ahsaini et al. presented a case of pure choriocarcinoma in a young man, revealed by jaundice. Despite general chemotherapy, he died due to lymph node metastasis at the hepatic hilum. ${ }^{18}$

There are a few case reports of the renal spread of choriocarcinoma in males. Li et al. reported the case of a 25-year-old man who presented with spontaneous renal hemorrhage, which on further workup was found to be secondary to choriocarcinoma; however, the case was complicated by cryptorchidism and hypospadias. ${ }^{3}$ The authors suggested choriocarcinoma was a difficult diagnosis because of its unusual presentation. We report on the second patient to be found with this presentation, who also had widespread disease with metastases in the lungs, mediastinal lymphnodes, and lower pole of the right kidney. His tumor marker levels of $\beta$-HCG were immensely high, corroborating the enormous tumor burden in the body. The lesion, however, showed significant reduction after chemotherapy. Lal et al. reported a case of choriocarcinoma in a female patient who presented with spontaneous renal hemorrhage. ${ }^{19}$

$\beta$-HCG has an important diagnostic and prognostic value in choriocarcinoma. ${ }^{5}$ The proportion of patients with elevated serum $\beta$-HCG levels is very high (96.4\%). ${ }^{4,5} \beta$-HCG levels are directly proportional to the tumor burden inside the body, and the fall in its levels is used to monitor the response and recurrence of the disease. $\beta$-HCG of $>1,000 \mathrm{mIU} / \mathrm{mL}$ is often considered diagnostic of choriocarcinoma. Our patient had $\beta$-HCG $>110,000 \mathrm{mIU} / \mathrm{mL}$. After a thorough search of the literature, we conclude this is the highest reported level to date. The patient in this case report underwent right inguinal orchiectomy followed by chemotherapy with BEP. After surgery, there was a temporary increase in $\beta-\mathrm{HCG}$, probably due to surgical manipulation, but the levels fell drastically after two cycles of chemotherapy.

This case report highlights the rarity of renal metastases in testicular choriocarcinoma, and we hope that the reporting of such a case will contribute to the data on this rare malignancy. Like other reported cases, our patient showed a very good response with chemotherapy. Most of the complications in patients with choriocarcinoma are due to chemotherapyrelated hemorrhages in tumor masses, but the increasing literature allows us to provide prophylactic management for expected complications and, therefore, improve rates of patient survival. $\square$
1. Eble JN, Sauter G, Epstein JI, Sesterhenn IA. World Health Organization Classification of Tumours. Pathology and genetics of tumours of the urinary system and male genital organs. Lyon: IARC Press; 2004. Available at: https://campatho.files.wordpress com/2009/05/who-of-urinary-system-and-male-genital-organs. pdf (accessed November 15, 2020).

2. Ro R, Amin M, Kim K, et al. Tumors of the male genital tract. In: Fletcher CDM (ed). Diagnostic Histopathology of Tumors, Philadelphia: Elsevier, 2007:827-8.

3. Li Y, Chen $\mathrm{G}$, Chen $\mathrm{H}$, et al. Spontaneous renal hemorrhage secondary to choriocarcinoma in a man with congenital hypospadias and cryptorchidism: a case report and literature review. BMC Cancer. 2018;18:543.

4. Jiang $F$, Xiang $Y$, Feng $F$, et al. Clinical analysis of 13 males with primary choriocarcinoma and review of the literature. Onco Targets Ther. 2014:7:1135-41.

5. Alvarado-Cabrero I, Hernández-Toriz N, Paner GP. Clinicopathologic analysis of choriocarcinoma as a pure or predominant component of germ cell tumor of the testis. Am J Surg Pathol. 2014;38:111-8.
6. Arana S, Fielli M, Gonzalez A, et al. Choriocarcinoma syndrome in a 24-year-old male. JRSM Short Rep. 2012:3:44.

7. Zeitjian VA, Arslan W, Borja-Alvarez A, Amar S. Choriocarcinoma syndrome: a potentially fatal complication of testicular cancer. Case Rep Oncol Med. 2019;2019:4092941.

8. Chaar A, Mouabbi JA, Alrajjal A, Barawi M. Metastatic testicular choriocarcinoma: an unusual cause of upper gastrointestinal bleed. Cureus. 2019;11:e5243.

9. Yokoi K, Tanaka N, Furukawa K, et al. Male choriocarcinoma with metastasis to the jejunum: a case report and review of the literature. J Nippon Med Sch. 2008;75:116-21.

10. Lowe K, Paterson J, Armstrong S, et al. Metastatic testicular choriocarcinoma: a rare cause of upper $\mathrm{Gl}$ bleeding. ACG Case Rep J. 2015;3:36-8.

11. Lauro $S$, Righini $R$, Onesti $C E$, et al. Gastric metastases from testicular cancer. case report and review of literature. $J$ Gastrointest Cancer. 2014:1:22-4.

12. Vardaros $M$, Subhani M, Rizvon $K$, et al. A Case of gastrointestinal bleeding due to duodenal metastasis from a testicular choriocarcinoma. J Gastrointest Cancer. 2013;44:234-7.
13. Senkal HA Y Ylmaz T Sözeri AB. Metastatic choriocarcinoma: a rare presentation as a neck mass. Ear Nose Throat J. 2013;92:E42.

14. Ahmadi SA, Tavakoli $H$, Samadi N. Neck mass as the first presentation of testicular choriocarcinoma. Eur Arch Otorhinolaryngol. 2006;263:290-2.

15. Toberer F, Enk A, Hartschuh W, Grüllich C. Testicular choriocarcinoma with cutaneous metastasis in a 19-year-old man. J Cutan Pathol. 2018;45:535-8.

16. Dotchin $\mathrm{S}$, Lakosha $\mathrm{H}$, Heathcote JG, Dickinson J. Uveal metastasis from testicular choriocarcinoma presenting as scleritis. Can J Ophthalmol. 2009;44:210-1.

17. Guber I, Zografos L, Schalenbourg A. Choroidal metastases in testicular choriocarcinoma, successful treatment with chemo- and radiotherapy: a case report. BMC Urol. 2011;11:24

18. Ahsaini M, Tazi F, Mellas S, et al. Pure choriocarcinoma of the testis presenting with jaundice: a case report and review of the literature. J Med Case Rep. 2012;6:269.

19. Lal A, Singhal M, Kumar $S$, et al. Bilateral renal and jejunal metastasis of choriocarcinoma presenting as spontaneous renal hemorrhage. Cancer Imaging. 2009;9:56-8. 